\title{
Ras gene mutation in acute myeloid leukemia and Myelodysplastic syndromes: a meta-analysis of its occurrence and prognostic relevance
}

\author{
Cheng Jingru ${ }^{1, a}$, Wang peng ${ }^{1, b}$, Zhang Tai ${ }^{1, \mathrm{c}}$, Yang Jinwei ${ }^{2, \mathrm{~d}}, \mathrm{Ma} \mathrm{Wei}^{3, \mathrm{e}}$, Li \\ Liyan $^{3, f}$, Guo Jianhui ${ }^{2, g}$ \\ ${ }^{1}$ Medical Faculty, Kunming University of Science and Technology, Kunming 650500, Yunnan, China. \\ ${ }^{2}$ Second Department of General Surgery, First People's Hospital of Yunnan Province, Kunming \\ 650032, Yunnan, China \\ ${ }^{3}$ Institute of Neuroscience, Kunming Medical University, Kunming 650500, Yunnan, China \\ achengjingru89@foxmail.com, bwp2010.cool@foxmail.com, ${ }^{\mathrm{C}}$ zhangtai1991417@163.com, ${ }^{\mathrm{d}}$ yangjin \\ wei800908@163.com, ${ }^{\mathrm{e}}$ mawei343897745@163.com, ${ }^{\mathrm{f}} \mathrm{kmliyanl@163.com,}{ }^{9}$ guojianhuikm@163.co
} $\mathrm{m}$

Keywords: Ras; mutation; Acute myeloid leukemia; Myelodysplastic syndromes; Prognostic

\begin{abstract}
Objective: The literature remains controversial on ras gene mutation prognostic significance for survival in AML and MDS. In this paper, study meta-analysis of its occurrence and prognostic relevance in acute myeloid leukemia and Myelodysplastic syndromes. Methods: The PubMed, Cochrane Library, EBSCO, and EMBASE databases were systematically searched for reports published from 1990 to 2015. Two authors independently assessed the methodological quality and the extracted data. The Hazard ratios and adjusted hazard ratios (HRs), a sensitivity analysis, and the publication bias were analyzed using the CMA v2 (Comprehensive Meta Analysis Version 2) software program. Results: Combined HRs suggested that an abnormal ras status had a different impact on survival: in AML group the HRs was 1.10 (0.97-1.26, $\mathrm{p}=0.15)$, was 1.89 (95\% CI, 1.56-2.30, $\mathrm{p}<0.001$ ) in MDS group. Further subtype of ras analysis, the pooled HRs in AML and MDS harbored Nras mutations were 1.34 (1.09-1.65, $\mathrm{p}=0.006)$ and $2.01(1.52-2.66, \mathrm{p}<0.001)$, respectively. The pooled HRs was $2.16(1.13-4.10, p=0.02)$ in AML patients with Kras. Conclusions: Take no account of the type of ras, the study found ras gene mutations seem not to correlate with the prognosis of patients with AML, however, further research subtype of ras, there was a poor prognosis in AML and MDS patients with Nras and AML patients with Kras. The association remains to be confirmed with a more precise analysis of a large sample and more studies should be focus on studying the prognosis between AML and MDS patients and the subtype of ras even special codon mutation.
\end{abstract}

\section{Introduction}

Acute myeloid leukemia (AML) and myelodysplastic syndromes (MDS) are characterized by a series of cytogenetic aberrations and mutations genes tightly connected with the leukemogenic process[1]. A prominent characteristic of MDS is its genetic instability, and nearly $30 \%$ of MDS cases will develop into AML[2][3][4]. In addition, AML and MDS that occur commonly in older patients, and the diagnostic median age is 67 years (54\% diagnosed age at 65 years or older) [5]. Although some treatments had improved the quality life of patients, the prognosis value was still very poor. According to Surveillance, Epidemiology and End Results data, the five-year survival rates from 2007 to 2012 was 26\% for AML patients [6]. Therefore, to find the prognosis factors seem to be much important for the patients with AML and MDS.

Ras oncogenes encode a family of membrane-associated proteins that regulate signal transduction on binding to a variety of membrane receptors. There are 3 functional ras genes-Nras, Kras, and Hras - and all homologs carry mutations nearly exclusively in codons 12, 13, and 61, conferring constitutive activation of the RAS protein, which subsequently is held in GTP-bound status[7]. Ras mutations have been described in various solid tumors and in hematologic 
malignancies[7]. Nras mutations seem to be the most prominent ras mutations in patients with AML and MDS[8]. Reuter et al found that transforming noncanonical Nras mutations at codon 60 and Kras mutations at codons 14, 74, and 146 were detected in primary leukemia samples, indicating that the prevalence of activating ras mutations may be underestimated in leukemia[9].

Although the high frequency of ras mutations was detected in patients with AML and MDS, the overall prognostic impact of mutations in patients with AML and MDS is controversial. Some investigators reported that ras mutations were associated with an adverse outcom[10], others have noted no significant prognostic impact[10]. Most previous studies didn't consider the subtype of ras, such as Nras, Kras or Hras and we suppose that this may be one reason that results in controversial. To demonstrate our supposition and clarify the relationship between ras gene mutations and the prognosis for patients with AML and MDS, it is necessary to perform a systematic review and meta-analysis of the published studies to derive a more precise estimation.

\section{Methods}

The results of a study were considered significant if the p-value for the statistical test comparing the survival distribution between the groups with and without abnormalities in the status of ras was $<0.05$. The study was then called "positive" (which meant that alteration was a poor prognosis for survival). If authors reported a significant result in a subgroup analysis only, the study was also considered to be positive. In addition, a study was called "negative" which reporting non-significant results or identifying ras gene mutations as associated with better survival. With regard to the quality scores, the scores of the studies were measured by the mean, standard deviation and range. Nonparametric tests were used to compare the distribution of the quality scores according the Mann-Whitney U test.

The effect of ras gene mutations on OS was measured by the pooled HR and 95\% CI. For each trial, this HR was estimated by a method depending on the results provided in a previous publication[11]. If articles did not provide the HR, the numbers of patients at risk in each group, and the logrank statistic or its p-value were used to allow for an approximate calculation of the HR. If the only exploitable data were in form of graphical representations of the survival distributions, the data from Kaplan-Meier survival curves was read by Engauge Digitizer version 2.11, and the minimum and maximum follow-up periods were obtained from the articles[12]. The statistical heterogeneity within studies was evaluated using a $\chi 2$-based Cochran's $Q$ statistic[13] and was further quantified using I2 statistics (I2 $=0-25 \%$, no heterogeneity; I2 $=25-50 \%$, moderate heterogeneity; I2 = 50-75\%, large heterogeneity; and I2 $=75-100 \%$, extreme heterogeneity. For values of I $2 \leqslant 50 \%$, the fixed-effects model was used; I $\geqslant 50 \%$, the random-effects model was used) [13]. 


\section{Results}

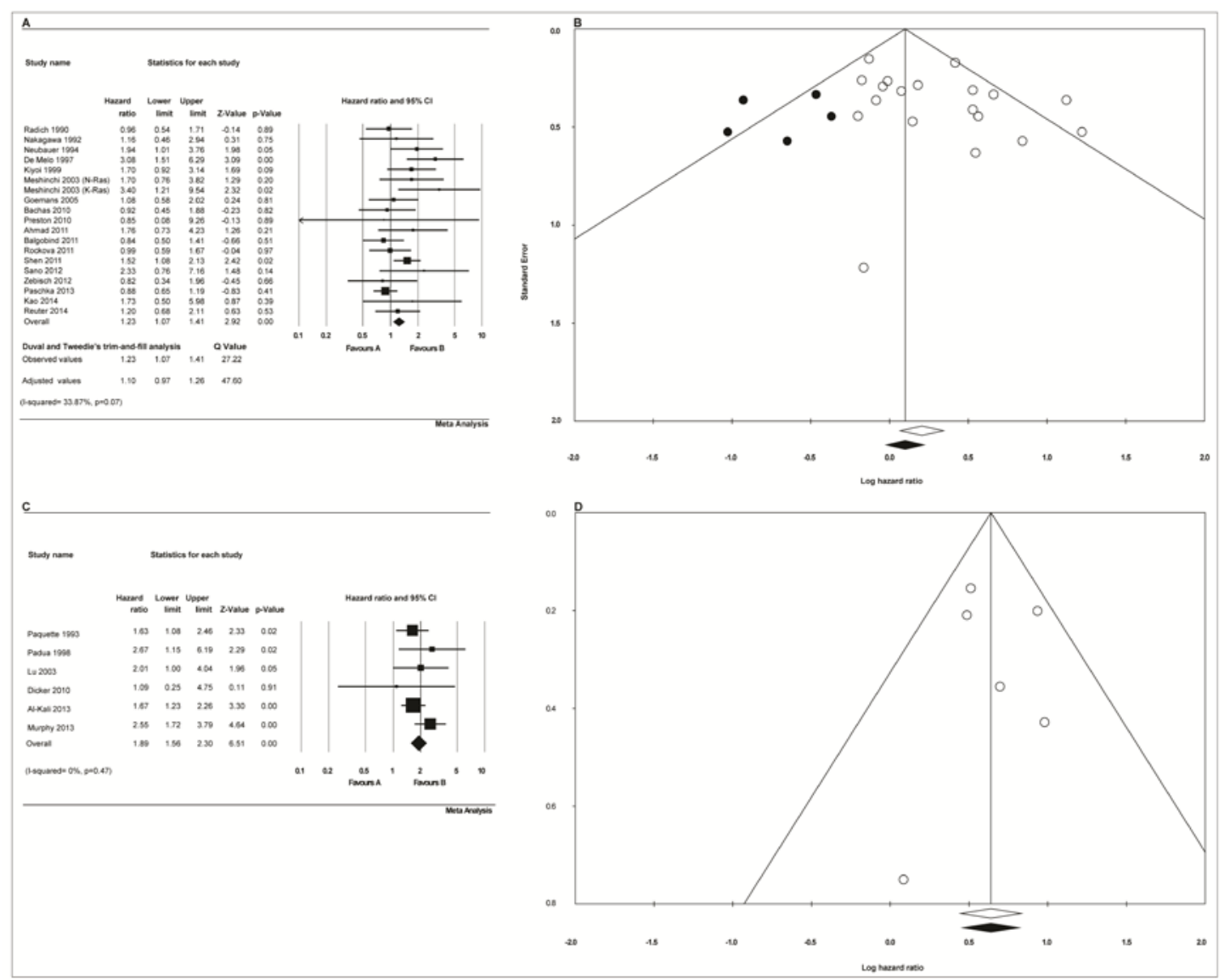

Meta-analysis

Fig.1 The Hazard ratio estimates of the prognosis of AML and MDS patients harbored with Ras mutation.

(A) A forest plot of the OS in AML patients harbored with Ras mutation. (B) Duval and Tweedie' $s$ trim-and-fill funnel plots were used to observe and adjust the publication bias in AML group. (C) A forest plot of the OS in MDS patients harbored with Ras mutation. (D) Duval and Tweedie' $s$ trim-and-fill funnel plots were used to observe and adjust the publication bias in MDS group. Among Fig B and D, The white circles represent observed studies, and black circles represent possibly missed studies imputed using Duval and Tweedie's trim-and-fill method. White and black rhombuses represent observed and theoretical combined effect size, respectively.

AML group. 18 studies (75\%) independently researched the relationship between ras mutation and the prognosis with AML patients. The pooled HR for the association between ras gene mutations and the OS in patients with AML was 1.10 (95\% CI, 0.97-1.26, p=0.15), with moderate heterogeneity between studies $(\mathrm{I} 2=33.87, \mathrm{p}=0.07)$. It was determined that ras gene mutations seem not to correlate with the prognosis of patients with AML (Fig.1A). A funnel plot identified the presence of publication bias, and Duval and Tweedie's trim-and-fill analysis was used to adjust the HR (Fig.1B), with $p=0.15$ in the Begg rank correction test and $p=0.11$ in Egger's linear regression test.

MDS group. 6 studies (25\%) independently researched the relationship between ras mutation and the prognosis with MDS patients. The pooled HR for the association between ras gene mutations and the OS in patients with MDS was 1.89 (95\% CI, 1.56-2.30, $\mathrm{p}<0.001$ ), without heterogeneity between studies $(\mathrm{I} 2=0, \mathrm{p}=0.47)$. It was determined that ras gene mutation correlate with the poor prognosis of patients with MDS (Fig.1C). A funnel plot identified the presence of publication bias, and Duval and Tweedie's trim-and-fill analysis was used to adjust the HR (Fig.1D), with p=0.57 in the Begg rank correction test and $\mathrm{p}=0.88$ in Egger's linear regression test. 


\section{Discussion}

Clinical heterogeneity is reflected by genetic findings with trisomy 8 and 22, KIT, and FLT3 mutations representing prognostic markers[14]. However, ras gene mutations as a prognostic factor for AML and MDS remain inconclusive. To our knowledge, this study is the first meta-analysis that explored the independent prognosis between ras gene mutations and patients of AML and MDS. Identification of independent prognostic factors allowed for the definition of high-risk groups of patients for whom specific therapy could be designed or a stratification in randomized trials performed. So far, for AML and MDS, useful prognostic factors are specific cytogenetic abnormalities, specific molecular markers, and percentage of marrow blasts and severity of cytopaenias[3] [15].

We extracted twenty-four studies that included 717 mutation cases among 5,704 patients. Nine sets of data indicated that ras gene mutations were associated with poor prognosis for patients with AML or MDS. Fifteen sets of data indicated that not any statistically significant association between ras gene mutations and OS. Based on our systematic review of literature with meta-analysis shows that ras gene mutations seem not to correlate with the prognosis of patients with AML, but exists a poor prognosis of patients with MDS if take no account of the type of ras mutations. In subgroup analysis, the poor prognostic value of AML is observed in Asia region, ONH method and publication year before 2000. In addition, all prognostic value of MDS is poor. Summarily, based on our subgroup analysis, the pooled HR may be affected by study regions, detection methods and year of publication. However, the relatively small sample size may not have enough statistical power to detect the association between ras gene mutations and survival and the results still need to be confirmed by an adequately designed prospective study and the exact value of an ras alteration needs to be determined by an appropriate multivariate analysis, taking into account the classical well-defined prognostic factors for AML and MDS.

\section{Summary}

To conclude, we demonstrated that our previous supposition that take no account of the type of ras may be one reason that results in controversial. Take no account of the type of ras, in the present systematic review with meta-analysis, ras mutations seem not to correlate with the prognosis of patients with AML but a poor prognostic value for survival in patients with MDS, however, further research subtype of ras, we confirmed that there was a poor prognosis in AML patients with Nras and Kras and an unfavorable impact on survival in MDS patients with Nras. This observation requires further investigation with an adequate statistical methodology to determine its exact place among the other known survival prognostic factors for AML and MDS and more studies should be focus on studying the prognosis between AML and MDS patients and the subtype of ras even special codon mutations.

\section{Acknowledgments}

This work was supported by the Natural Science Foundation of China (No. 81260075)

\section{References}

[1] Germing U, Haferlach T. Diagnostics of myelodysplastic syndromes (MDS) and acute myeloid leukemia (AML)[J]. Laboratoriumsmedizin-journal of Laboratory Medicine, 2015, 39(5):291-299.

[2] Ozcan MA, Ilhan O, Ozcebe OI, Nalcaci M, Gulbas Z. Review of therapeutic options and the management of patients with myelodysplastic syndromes. Expert Rev Hematol 2013;6:165-189.

[3] Lionel A, Raphael I, Pierre F. Myelodysplastic syndromes.[J]. Lancet, 2014, 383(9936):97-109.

[4] Fenaux P, Haase D, Sanz G F, et al. Myelodysplastic syndromes: ESMO Clinical Practice Guidelines for diagnosis, treatment and follow-up.[J]. Annals of Oncology Official Journal of the 
European Society for Medical Oncology, 2014, 25 Suppl 3(9):57-69.

[5] Tinsley S M. A Comparison of Quality of Life between Intense and Non-Intense Treatment for Patients with Acute Myeloid Leukemia and High-Risk Myelodysplastic Syndrome[J]. Dissertations \& Theses - Gradworks, 2015.

[6] Surveillance, Epidemiology and End Results (SEER) Program Cancer Statistics Review 1975-2012. [Internet]. 2015, April 1 -. 4 Available from: http://seer.cancer.gov/csr/1975_2012/results_merged/sect_13_leukemia.pdf\#search=five

[7] Berman J N, Gerbing R B, Alonzo T A, et al. Prevalence and clinical implications of NRAS mutations in childhood AML: a report from the Children's Oncology Group.[J]. Leukemia Official Journal of the Leukemia Society of America Leukemia Research Fund U K, 2011, 25(6):1039-1042.

[8] Gautam Borthakur MD, Leslie Popplewell MD, Michael Boyiadzis MD, et al. Activity of the oral mitogen-activated protein kinase kinase inhibitor trametinib in RAS -mutant relapsed or refractory myeloid malignancies[J]. Cancer, 2016.

[9] Reuter C W M, Jürgen K, Onono F O, et al. Lack of noncanonical RAS mutations in cytogenetically normal acute myeloid leukemia.[J]. Annals of Hematology, 2014, 93(6):977-982.

[10]Kadia T M, Hagop K, Steven K, et al. Clinical and proteomic characterization of acute myeloid leukemia with mutated RAS.[J]. Cancer, 2012, 118(22):5550-5559.

[11]Ren J, Li G, Ge J, et al. Is K-ras gene mutation a prognostic factor for colorectal cancer: a systematic review and meta-analysis.[J]. Diseases of the Colon \& Rectum, 2012, 55(55):913-23.

[12]WANG, P., LIU, H., JIANG, T. \& YANG, J. 2015. Cigarette Smoking and the Risk of Adult Myeloid Disease: A Meta-Analysis. PLoS One, 10, e0137300.

[13]BOWDEN, J., TIERNEY, J. F., COPAS, A. J. \& BURDETT, S. 2011. Quantifying, displaying and accounting for heterogeneity in the meta-analysis of RCTs using standard and generalised Q statistics. BMC Med Res Methodol, 11, 41.

[14]Peter P, Juan D, Schlenk R F, et al. Secondary genetic lesions in acute myeloid leukemia with inv(16) or t(16;16): a study of the German-Austrian AML Study Group (AMLSG).[J]. Blood, 2013, 121(1):170-177.

[15]NCCN. 2014. (NCCN) Clinical Practice Guidelines in Oncology: Acute Myeloid Leukemia (Version 1.2015) [Online]. Available: http://www.nccn.org/professionals/physician_gls/pdf/aml.pdf. 